\title{
Sagesse proverbiale à l'usage d'une cour : le cheval de Jeunesse, le rocher de Fortune
}

Jean-Claude Mühlethaler

\section{(2) OpenEdition}

1 Journals

Édition électronique

URL : http://journals.openedition.org/edl/1380

DOI : $10.4000 /$ edl. 1380

ISSN : 2296-5084

Éditeur

Université de Lausanne

\section{Édition imprimée}

Date de publication : 1 décembre 2018

Pagination : 151-174

ISBN : 978-2-940331-69-7

ISSN : 0014-2026

\section{Référence électronique}

Jean-Claude Mühlethaler, « Sagesse proverbiale à l'usage d'une cour : le cheval de Jeunesse, le rocher de Fortune », Études de lettres [En ligne], 3-4 | 2018, mis en ligne le 15 décembre 2020, consulté le 17 décembre 2020. URL : http://journals.openedition.org/edl/1380 ; DOI : https://doi.org/10.4000/edl. 1380

\section{(c) Études de lettres}




\section{SAGESSE PROVERBIALE À L'USAGE D'UNE COUR: LE CHEVAL DE JEUNESSE, LE ROCHER DE FORTUNE}

Dans la salle dite "des conférences» du château Saint-Maire se trouve une peinture qui n'a guère retenu l'attention des spécialistes. On y voit une femme nue, les cheveux au vent, assise sur un cheval fou en train de galoper vers le rocher de Fortune, ainsi que le précise le quatrain qui accompagne la représentation. Or, un dessin du magnifique recueil (BnF, fr. 24461) commandité par la puissante famille des Robertet, dont les membres ont servi les ducs de Bourbon et le roi de France, offre le pendant exact de la peinture voulue par Aymon de Montfalcon. Une lecture croisée des deux témoins permet d'en dégager les enjeux idéologiques convergents et d'établir, en élargissant l'enquête, des liens que le prince-évêque de Lausanne entretenait avec d'autres foyers culturels. Un esprit d'époque, celui d'une élite entre Moyen Âge et Renaissance, flotte au château Saint-Maire de Lausanne.

Ainsy dont qu'en Jeunesse estoye Sans tenir rigle ne compas ${ }^{1}$.

Prince-évêque, ambassadeur, mécène, Aymon de Montfalcon fut aussi "poète amateur en sa jeunesse ${ }^{2}$. À elles seules, les peintures du corridor central du château Saint-Maire à Lausanne fournissent une preuve éclatante de sa curiosité intellectuelle: le Breviaire des nobles, dû à la plume d'Alain Chartier, secrétaire du futur roi Charles VII, et qui fut le livre de chevet des nobles aux $\mathrm{XV}^{\mathrm{e}}$ et $\mathrm{XVI} \mathrm{I}^{\mathrm{e}}$ siècles, y fait face aux «Enseignes" des Douze Dames de Rhétorique, œuvre de George Chastelain, grand

I. Michault Taillevent, "Le Passe Temps», v. 71 sq., in Un poète bourguignon du $X V^{e}$ siècle, éd. R. Deschaux, p. 137.

2. F. Féry-Hüe, "Aymon de Montfalcon», p. 119 sq. 
indiciaire de la cour de Bourgogne et figure de proue de ceux qu'on a appelés les "grands rhétoriqueurs". L'évêque fait dialoguer, dans son "corridor-codex" ${ }^{3}$, les textes phares des deux cours rivales dont il dépasse l'antagonisme en suivant la démarche de Chastelain qui n’a cessé de $s^{\prime}$ inspirer (mais sans jamais l'avouer! ${ }^{4}$ ) de l'œuvre de Chartier. Dans un geste d'appropriation, il les place sous sa devise personnelle, d'origine virgilienne (Æn. I, 18) : Si qua fata sinant, répété tout au long de la frise, traduit l'ambition du prince de réaliser à sa cour l'idéal à la fois éthique et rhétorique que transmettent les deux poèmes.

Nous ne reviendrons ici que brièvement sur les décorations du corridor $^{5}$, préférant nous pencher sur une représentation que la critique a largement négligée. La peinture en question se trouve dans la niche à droite de la fenêtre axiale de ce qu'on appelle, faute de mieux, la "salle des conférences», située elle aussi au rez-de-chaussée (côté nord). Comme le programme iconographique du corridor, la peinture témoigne des liens que le prince-évêque de Lausanne entretenait avec d'autres foyers culturels. Qu'y voit-on? (fig. 1) Une femme nue, les cheveux au vent, est assise sur un cheval, sans bride ni selle, en train de galoper vers ce qui est, nous le verrons, un rocher. "On a cru», est-il écrit sur le site du Patrimoine Vaudois ${ }^{6}$, "à une allégorie de la Vérité». Heureusement, l'énoncé laisse planer le doute: en effet, une femme nue ne représente pas nécessairement la Vérité, ni d'ailleurs Vénus, incarnation de la luxure au Moyen Âge. Il suffisait pourtant de suivre la même démarche qu'exige le décodage des peintures dans le corridor, c'est-à-dire de lire les vers transcrits dans le phylactère - lointain héritier des versus ad scripturas carolingiens - pour comprendre que le message est de tout autre nature:

3. Dans son étude consacrée aux Storie al muro, M. L. Meneghetti intitule le chapitre aux espaces peints des demeures seigneuriales: "Camera illa codex est». Une formulation des plus heureuses!

4. Sur la relation entre Chastelain et son maître inavoué, voir E. Doudet, Poétique de George Chastelain (1415-1475), p. 705-711.

5. À leur sujet, voir M.-R. Jung, "Les "Douze Dames de Rhétorique” "; K. Straub, " "Se riens y a qui soit de noble umbrage" "; J.-C. Mühlethaler, "Ein dichterisches und politisches Manifest in Lausanne». Un mémoire de l'Université de Genève leur a été consacré: L. Rochat, Les peintures du corridor du château Saint-Maire à Lausanne.

6. <http://www.patrimoine.vd.ch/accueil/chateau-saint-maire/le-chateau>. 


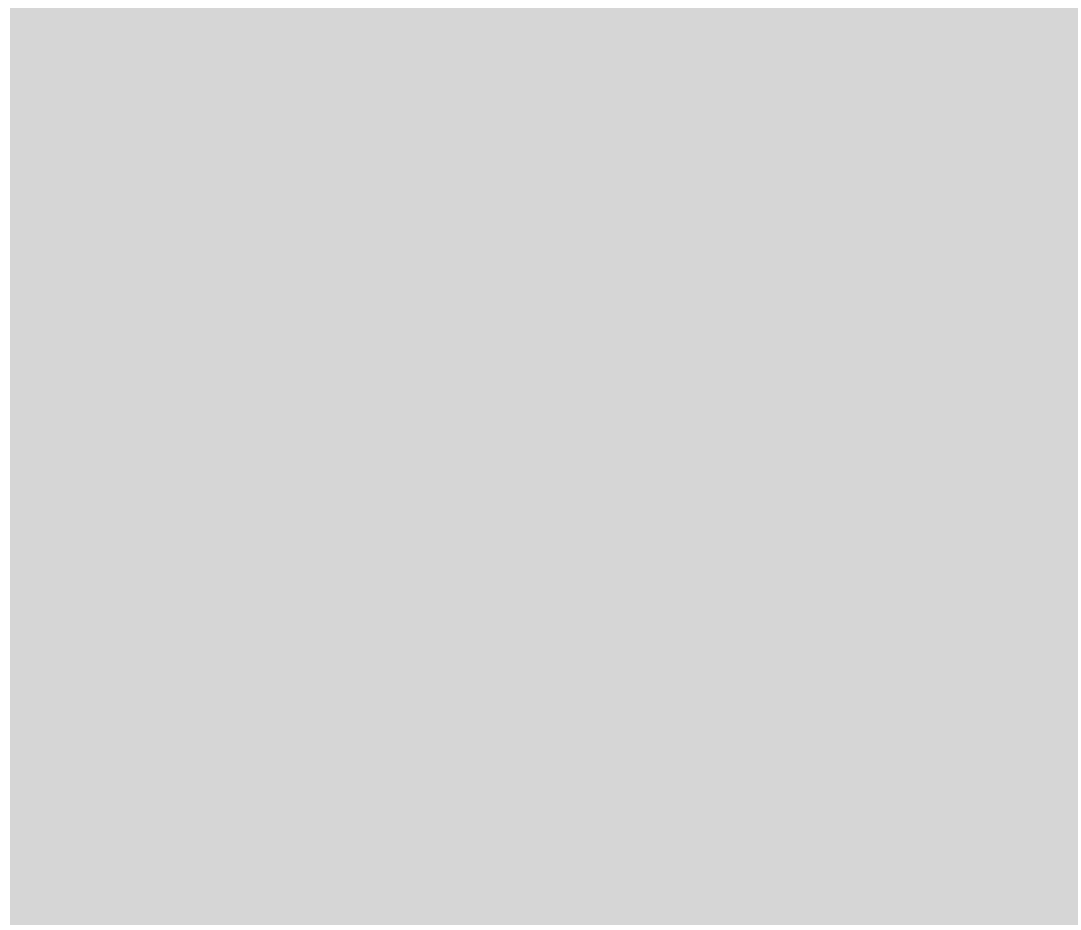

Fig. 1 - Jeunesse et le rocher de Fortune, peints dans une niche de la salle des conférences du château Saint-Maire à Lausanne. Photographie Rémy Gindroz, 2018.

[Tan]t fier cheval sans selle ne sans frain $(\ldots \ldots \ldots)$ jour et nuit la traverse Povre Jeunesse en peril inhumain Contre le roch de Fortune diverse*. *hostile; variable

L'inscription appelle plusieurs remarques. Commençons par relever qu'il s'agit d'un quatrain (décasyllabique), forme qui, du XIII ${ }^{\mathrm{e}}$ au XVI ${ }^{\mathrm{e}}$ siècle, sert souvent de moule à des textes moralisateurs ou sentencieux. De cette poétique du docere témoigne notamment le Testament (en quatrains d'alexandrins monorimes) attribué à Jean de Meun et largement diffusé par le manuscrit ${ }^{7}$, puis grâce à l'imprimerie. Considéré comme une œuvre de vieillesse, le Testament prend la forme d'un acte de contrition

7. Au sujet de ce texte, voir S. Buzzetti Gallarati (a cura di), Jean de Meun, Testamento e Codicillo. 
doublé d'une renonciation aux écrits de jeunesse (le Roman de la Rose), jugés désormais vains et futiles, car - trop voués à la delectatio - ils négligeaient l'utilitas. L'image d'une Jeunesse irréfléchie et irresponsable, emportée par un cheval non maîtrisé, telle qu'elle s'offre à nous dans la salle des conférences, fait écho à la critique exprimée en ouverture du Testament. Elle s'inscrit, plus généralement, dans une tradition moralisatrice qui, $s^{\prime}$ inspirant de versets bibliques ${ }^{8}$, met en garde contre les tentations auxquelles l'homme est exposé dans sa jeunesse quand il ne quitte que trop facilement le droit chemin. C'est là, il faut bien l'avouer, un lieu commun qui, dans sa banalité passe-partout, est proche d'une sagesse proverbiale. Plus que le contenu, c'est donc le choix des métaphores, de nature visuelle, propres à frapper l'esprit, qui interpelle.

Le "fier cheval», c'est-à-dire le cheval sauvage qui emporte Jeunesse n'est pas sans rappeler le fougueux cheval d'Orgueil, issu de la Psychomachie de Prudence, qui finit par jeter son cavalier à terre dans la tradition iconographique et littéraire médiévale 9 . Les connotations négatives du cheval - animal pourtant emblématique de la chevalerie - se rencontrent déjà dans la Bible: il y est l'expression de l'orgueil humain, l'intellectus (Ps. 31, 9) lui fait défaut et c'est un animal particulièrement luxurieux (Jér. 5, 8-9), ce que relèvent également Aristote et, dans son sillage, les encyclopédies médiévales ${ }^{10}$. La scène du château Saint-Maire s'éclaire à la lumière de la tradition qui fait du cheval le symbole de la meretricia procacitas ${ }^{11}$ : soumise à l'empire des sens, incapable de les mâ̂triser, Jeunesse paie au prix fort sa quête effrénée de plaisirs. L'image de la nudité du péché, telle qu'on la connaît de la Bible, vient à l'esprit, l'idée de la luxure aussi, associée à la jeunesse chez Ézéchiel (Éz. 23, 21); mais la nudité renvoie ici avant tout à la vulnérabilité (pour ne pas dire à l'innocence) de la Jeunesse, à son manque de préparation face aux épreuves de la vie. La tendre jeune fille emportée par son coursier suscite en effet la pitié: les adjectifs "povre» et «inhumain» sont révélateurs à cet égard, car ils laissent transparaître l'empathie du locuteur

8. On pensera aux conseils de l'Ecclésiaste au jeune homme (Eccl. 12) ou à la fin misérable d'Absalom révolté contre son père David (II Sam. 18). Voir aussi Eccl. 11, 8-10; Ps. 25, 7; Jér. 31, 19; Lam. 3, 27; Éz. 23, 21.

9. Voir D. Hüe, "L'orgueil du cheval» et (aussi pour les remarques qui suivent) J.-C. Mühlethaler, Fauvel au pouvoir, p. 64-68.

IO. B. Prévot, B. Ribémont, Le cheval en France au Moyen Âge, p. 283.

II. G. de Tervarent, Attributs et symboles dans l'art profane, p. 118. 
(implicite), homme apparemment sage et avisé qui prévoit l'issue fatale de la folle chevauchée. Une attitude aussi bienveillante est inhabituelle chez les moralistes - il suffit d'ouvrir le chapitre dans lequel Christine de Pizan "se plaint de jeunesse»! ${ }^{12}$ - et se lit comme une note personnelle d'Aymon de Montfalcon. Si tel est bien le cas, la présence des armoiries de l'évêque, surmontées de la mitre, au plafond de la niche, n'est pas due au hasard. Comme dans le corridor où sa devise trône au-dessus des textes d'Alain Chartier et de George Chastelain, le prince-prélat s'approprie ici le quatrain, fort de l'autorité morale que lui confère sa dignité ecclésiastique. Mais la représentation de la salle se distingue dans la mesure où l'évêque fait ici figure de locuteur, voire d'auteur, puisque par les armoiries, il est la seule instance à laquelle le quatrain peut être rattaché. C'est à lui qu'on attribuera l'infléchissement de la leçon traditionnelle où perce une indulgence inattendue face aux errances de la jeunesse.

Ce qui attend la malheureuse Jeunesse, c'est le "roch de Fortune»! Moins souvent associé à la "diverse" (inconstante/hostile) déesse que sa célèbre roue, le rocher représente la dure réalité sur laquelle vient se briser la démesure de l'homme sourd à la voix de la raison. Par la mise en garde qu'il véhicule, le quatrain de la salle fait pendant ${ }^{13}$ au phylactère dans le dernier panneau de la paroi nord, à la fin du corridor central. Placé au-dessus de l'écusson triparti où sont représentés, dans la partie supérieure, le clergé et la noblesse et, dans la partie inférieure, le Tiers État (un paysan en train de labourer), il livre la senefiance de cette représentation traditionnelle de la société. Tous les hommes sont les descendants d'Adam et Ève:

Cet escu fait signifiance

Que nous fumes d'une naissance

Et par sagement maintenir

Peut petit homme haut venir.

Nous avons là l'envers et l'endroit de la même leçon. L'ascension sociale est possible, mais à condition qu'on suive la voie de la sagesse sans se

I2. Christine de Pizan, Le Livre de l'advision Cristine, p. 108 sq. À ce sujet, voir B. Ribémont, «Le regard de Christine de Pizan sur la jeunesse (à propos du Charles V)».

I3. Le rapprochement entre la décoration de la salle et les peintures du corridor a déjà été proposé par K. Straub, "Se riens y a qui soit de noble umbrage" ", p. 278 sq. 
laisser séduire par les appâts trompeurs du monde en succombant au péché d'orgueil, lequel pousse le «petit homme» à s'élever au-dessus de ce que permettent ses mérites et son origine sociale. Voilà peut-être pourquoi Adam et Ėve, qui tiennent l'écusson, sont habillés en bourgeois respectables, contrairement à la tradition qui les représente soit nus (avant la chute), soit en train de travailler la terre à la sueur de leur front (après avoir été chassés du paradis). En cette toute fin du Moyen Âge, l'ambition individuelle a donc droit de cité, elle peut même porter ses fruits pourvu qu'elle respecte le cadre imposé par une société habituellement méfiante face aux hommes nouveaux ${ }^{14}$, ces parvenus dont on ne soupçonne que trop volontiers la moralité.

La devise de l'évêque fait, elle aussi, écho à la leçon de la chambre. Si qua fata sinant (Æn. I, 18) laisse transparaître la crainte d'un homme qui se sait soumis à des forces qui le dépassent. Seulement, le registre change: au français s'oppose le latin, langue de l'érudition, à une morale proverbiale le modèle épique, à Fortune enfin les fata, auxquelles sont soumis, chez Virgile, le héros et les dieux eux-mêmes. En arrachant à leur contexte les mots de Junon rêvant un avenir glorieux pour Carthage, Aymon de Montfalcon affirme - au-delà d'une apparente humilité l'importance de ses propres projets. Il laisse percer ses ambitions politiques et culturelles face à ceux qui savent lire les indices disséminés dans le château. Contrairement à la folle Jeunesse, qui n'est guère qu'un lointain souvenir et dont la nudité - comme celle, symbolique, du cheval ${ }^{15}$ sans selle et sans bride - est incompatible avec la dignité d'un prince d'Église, notre évêque se présente sous les traits d'un homme sage et prudent, en marche vers l'honneur et la gloire. Aymon ne se place pas ou plus sous l'égide de l'inconstante Fortune, maîtresse des aléas de la vie et allégorie du hasard plutôt que ministre de la Providence divine ${ }^{16}$. Des abîmes séparent cette divinité de ce que George Chastelain appelle, en utilisant le terme forgé directement sur le latin, "fat et vielle

I4. Voir B. Bove, "Réflexions sur les hommes nouveaux et l'ascension sociale au Moyen Âge, de Leudaste à Jacques Cœur, en passant par Pareto".

I5. Agostino Paravicini a rappelé, lors de la discussion, que le cheval est considéré comme le double du souverain et se doit d'être bridé, sellé et paré aux couleurs du dignitaire qu'il porte.

I6. Le statut de Fortune est flottant au Moyen Âge, tour à tour fille de Dieu ou divinité aveugle et imprévisible dont les clercs nient parfois jusqu'à l'existence: voir J.-C. Mühlethaler, «Quand Fortune, ce sont les hommes». 
destinee " ${ }^{17}$. Le fatum, c'est ce qui est prédit et établi depuis toujours (il s'agit, chez Chastelain, de la vision apocalyptique de Childéric); la bien nommée "vielle destinee" se réalise fatalement un jour, que ce soit la fondation de Rome ou la chute de Carthage. De toute évidence, Aymon de Montfalcon se rêve une place dans les livres d'histoire; "muny d'honneur, de sens et de sçavoir " ${ }^{18}$ - comme le clame Antitus, son chapelain -, il se voit un destin sub specie aeternitatis.

La carrière du prince-évêque, homme mûr et avisé, ne s'est pas brisée contre le rocher de Fortune. Il est tentant de lire le contraste entre la salle de conférences et le corridor à la lumière de la vie d'Aymon de Montfalcon. À une jeunesse dédiée aux plaisirs et à la poésie courtoise aurait succédé l'engagement du diplomate dans les affaires de son temps et la gestion de l'évêché. Le passage d'une jeunesse frivole à la sagesse de l'homme responsable relève toutefois trop du topos pour être pris pour un pur aveu de nature autobiographique. Le Testament de Jean de Meun, déjà cité, qui s'ouvre sur le rejet des écrits antérieurs au nom d'une attitude plus morale, est un modèle du genre. Le Passe Temps de Michault Taillevent, auteur bourguignon au service de Philippe le Bon, lui emboîte le pas. Le temps des "complaintes» succède chez lui aux «virelais de flours" qu'il a rédigés alors qu'il était jeune ${ }^{19}$; il rejette la matière courtoise au nom d'une écriture morale, truffée de proverbes. «Telle plume, tel escripvain» (v. 91), conclut Taillevent, et c'est bien ce dont il s'agit: le changement de registre - du proverbial à l'épique et au latin pour Aymon de Montfalcon - est une posture littéraire ${ }^{20}$ où s'inscrit symboliquement le passage de la jeunesse à l'âge adulte. C'est le même mouvement, de la folie à la sagesse ou, plus précisément, du règne de Sensualité à celui d'Entendement, qui marque le Séjour d'Honneur d'Octovien de Saint-Gelais, évêque d'Angoulême. Le récit, dédié au roi

I7. George, Chastelain, Le Dit de Verité, p. 210, v. 548. Pour la vision de Childéric, attribuée par Chastelain à son père «Meronnee» (v. 545), voir la note à la page $174 \mathrm{de}$ l'édition.

I8. Antitus, La Satyre Megere, v. 77, in Poésies. Le vers se trouve dans les vers conclusifs du poème où se lit en acrostiche le nom de l'évêque: "Ayme de Monfalcon".

19. Michault Taillevent, "Le Passe Temps», v. 86 et 89 . Voir aussi la citation en exergue, tirée du même texte.

20. Sur cette notion, voir l'introduction théorique dans le volume dirigé par J.-C. Mühlethaler, D. Burghgraeve, Un territoire à géographie variable. 
Charles VIII, a la forme d'un pèlerinage de vie humaine ${ }^{21}$ qui retrace les errements de l'Acteur, c'est-à-dire d'un moi, mais d'un moi dans lequel chaque lecteur peut se reconnaître. Les enjeux autobiographiques restent secondaires dans cette œuvre aussi bien qu'au château, que ce soit à cause du poids de la tradition morale ou de la portée générale du message, assurée par le recours à l'allégorie et son cortège de personnifications. Les implications personnelles des peintures ne sont donc perceptibles qu'à un cercle d'initiés, liés d'amitié avec Aymon, tandis que les invités de passage à Lausanne y auront vu l'expression d'un idéal éthique et esthétique qui leur est familier. Le discours iconographique du château Saint-Maire est une mise en scène savamment orchestrée, laquelle offre au public l'image reluisante que le prince-évêque entend donner de lui-même en se coulant dans un moule connu, susceptible de parler à tous, mais qu'il adapte subtilement en prêchant - mine de rien - pour sa propre paroisse. Le poids de la tradition ou, plus précisément, les réminiscences littéraires s'invitent aussi dans la représentation de Jeunesse emportée vers le rocher de Fortune. Dans l'Anticlaudianus d'Alain de Lille ${ }^{22}$, que suit Jean de Meun dans le Roman de la Rose, la maison de Fortune se trouve sur un rocher en pleine mer, battu par les vents ${ }^{23}$ :

Une roche est en mer seanz

Bien parfont, el milieu leanz*, *là-dedans

Qui sus la mer en haut se lence*, $\quad{ }^{*}$ se dresse

Contre cui la mers grouce et tence*. * gronde et lutte

Li flot la hurtent et debatent*

Qui tous jours a li se combatent (... ${ }^{24}$.

Le mouvement de la mer, comme l'élan du cheval, se brise contre la fermeté du rocher. Dans les Dictz moraulx d'Henri Baude (nous y

2I. Voir Ph. Maupeu, Pèlerins de vie humaine, qui conclut le chapitre sur Octovien en rappelant qu'un récit allégorique n’a pas «la vocation de refléter le réel, du moins dans le sens le plus trivial du terme» (p. 589).

22. Citation et commentaire du passage, inspiré du De consolatione Philosophae de Boèce, chez Y. Foehr-Janssens, "La Maison de Fortune dans l'Anticlaudianus d'Alain de Lille», p. 136 sq.

23. Dans la satire anticuriale, cette description allégorique est appliquée (chez Pierre d'Ailly ou Eustache Deschamps) à la demeure du tyran: voir J.-C. Mühlethaler, «Le tyran à table», p. 51-55.

24. Le Roman de la Rose, v. 5917-5922. 
reviendrons), qui datent de la fin du $\mathrm{XV}^{\mathrm{e}}$ siècle, une rubrique dépeint une scène comparable, fournissant des indications pour une future tapisserie: " ung homme dedans ung navire qui va hurter contre un roq ouquel a deux seraines", telle est l'illustration qui devra accompagner le quatrain décasyllabique - encore un! - dénonçant les périls de la «mer / De ce monde» ${ }^{25}$ instable et trompeur. Le contemptus mundi, encore et toujours!

Les trois descriptions allégoriques se servent d'éléments figuratifs apparentés, plus ou moins interchangeables, à travers lesquels s'exprime le conflit entre mouvement et stabilité. Elles répètent la même mise en garde: le rocher de Fortune ou celui des sirènes est l'écueil contre lequel se brise la course de l'homme leurré par le vain éclat des biens et plaisirs éphémères. Mais, dans le Roman de la Rose, les vers qui suivent dépeignent le rocher tour à tour submergé par les flots et dominant la mer par beau temps. Le rocher lui-même "ne retient nulle forme" (v. 5929), car il est instable et changeant, à l'image de la déesse qui l'habite. Le message est sensiblement différent au château Saint-Maire: Fortune a beau être qualifiée de "diverse", elle n'est pas saisie dans ses métamorphoses, mais apparaît comme un obstacle qui se dresse, stable et redoutable, sur le chemin de la jeunesse. Aymon de Montfalcon fut, on le sait, un fervent lecteur de Jean de Meun ${ }^{26}$, mais sa source d'inspiration est à chercher ailleurs, plus près dans le temps et dans l'espace. La représentation de Jeunesse lui vient du même milieu auquel il doit de connaître les Douze Dames de Rhétorique.

Les Douze Dames de Rhétorique sont, rappelons-le ${ }^{27}$, le fruit d'échanges épistolaires qui eurent lieu, en 1463, entre Jean Robertet, secrétaire de Jean II de Bourbon, George Chastelain, au service de Philippe le Bon, et Jean de Montferrant, chambellan à la cour de Bourbon, qui servit d'intermédiaire entre les deux écrivains. Ce dernier était le frère de Benoît de Montferrand, le prédécesseur d'Aymon de

25. Henri Baude, Dictz moraulx pour faire tapisserie, p. 134 (v. 1-2).

26. Comme en témoigne le Dialogue entre le Gris et le Noir (BnF, fr. 25421), attribué à Aymon de Montfalcon (les lettres de son monogramme, ".M. et A», figurent au fol. $42 \mathrm{v}$ ), où l'on retrace aussi bien les souvenirs de la Belle Dame sans mercy d'Alain Chartier que la présence de Danger ou Malebouche, personnifications empruntées au Roman de la Rose.

27. Nous renvoyons à l'introduction de $\mathrm{D}$. Cowling à son édition des Douze Dames de Rhétorique. 
Montfalcon au poste d'évêque à Lausanne. Selon toute probabilité, il lui a fait connaître les Douze Dames qui, conservées dans des manuscrits richement illustrés, ne semblent guère avoir circulé hors des milieux curiaux ${ }^{28}$. N'oublions pas Antitus Favre, chapelain du prince-évêque vers 1500 et auteur de vers inspirés par George Chastelain (Le Portail du temple Bocace). Il avait d'abord été chapelain à la Sainte-Chapelle de Dijon, peut-être même au service des ducs Valois ${ }^{29}$ avant le rattachement du duché à la couronne de France en 1477. Les liens entre Lausanne et la Bourgogne sont multiples.

Les échanges entre ces foyers littéraires qu'étaient les cours princières au $\mathrm{XV}^{\mathrm{e}}$ siècle mériteraient une étude systématique. Ainsi, Jean Robertet, mais aussi son maître, Jean II de Bourbon, lui-même poète à ses heures, ont séjourné à Blois, où ils ont transcrit, le premier une ballade, le second dix rondeaux ${ }^{30}$ dans le manuscrit personnel de Charles d'Orléans. Jean Robertet est surtout l'auteur d'une adaptation en français des Triomphes ${ }^{31}$ de Pétrarque, qu'on retrouve dans un recueil de dessins servant de modèles pour des tapisseries. Nous le connaissons grâce à deux manuscrits: le manuscrit 5066 (conservé à la Bibliothèque de l'Arsenal), qui a appartenu à la famille Robertet, est une copie essentiellement fidèle ${ }^{32}$ du manuscrit de luxe ${ }^{33}(\mathrm{BnF}$, fr. 24461) destiné probablement à Charles III de Bourbon. Nous suivrons ici la version originale du recueil: elle est en partie de la main du fils aîné de Jean, François I ${ }^{\text {er }}$ Robertet ${ }^{34}$, notaire et secrétaire du duc de Bourbon qui était connétable

28. Voir la thèse fondamentale de K. Straub, "Les Douze Dames de Rhétorique” in Text und Bild, surtout p. 246-252.

29. La question est soulevée par É. Bousmar, «Faut-il restituer Antitus Favre à la littérature bourguignonne?», p. 11-13.

30. Voir l'index des noms dans Charles d'Orléans, Poetry of Charles d'Orléans and His Circle.

31. Jean Robertet, Euvres, p. 179-184.

32. Le manuscrit daterait de 1518: voir Les Proverbes illustrés, p. 40 sq. et, pour les différences entre les deux manuscrits, p. 15 sq. et 44.

33. Pour les remarques qui suivent, voir F. Buttay-Jutier, Fortuna, p. 332-335.

34. François Robertet est aussi le scribe du BnF, fr. 12490, exécuté probablement avant 1514. Il contient une transcription partielle des Douze Dames de Rhétorique, la Doleance de Megere de Regnaud le Queux (dont s'est inspiré Antitus Favre pour sa Satyre Megere), les Triumphes de Pétrarque par Jean Robertet, des pièces lyriques de Jean Molinet, François Villon (dont une ballade est adressée au duc de Bourbon, fol. 96r), Octovien de Saint-Gelais, Clément Marot, ainsi que Plusieurs Diz pour faire tapisserie faiz [par] l'esleu Baude (fol. 118r). 
de France (1515-1521) sous François I ${ }^{\text {er }}$, avant qu'il ne se révoltât contre son suzerain et passât au service de Charles Quint. Ce puissant seigneur est célébré au fol. 141r pour sa bravoure à la bataille d'Agnadel en 1509. C'est autour de cette date que le recueil, fait d'unités disparates ${ }^{35}$, aura été achevé (à l'exception de quelques ajouts postérieurs ${ }^{36}$ ) après une première phase de rédaction qui remonte à $1500-1505^{37}$. Il est donc de peu antérieur aux peintures du château Saint-Maire, exécutées probablement vers $1510^{38}$.

Le blason des Robertet ${ }^{39}$ (d'azur à la bande d'or, chargée d'un demivol de sable, accompagnée d'une étoile en chef de deux pointes) se trouve au fol. 115r, intégré à une scène de caractère mythologique. L'écu est suspendu à un arbre à proximité d'Hercule enchaîné et de Cupidon assoupi avec, pour arrière-fond, le mont Parnasse à gauche et la ville de Thèbes à droite. Il ne saurait être question de saisir ici dans sa complexité le dessin qui accompagne l'épigramme (Dormit Amor, pharetreque iacent, requiescit et arcus) dû à la plume d'un humaniste italien ${ }^{40}$. Contentonsnous de relever l'intérêt pour la matière antique, que les Robertet partagent avec Aymon de Montfalcon. Une esthétique comparable semble régner à la cour de Bourbon et à la cour de Lausanne: comme les peintures murales du château Saint-Maire, le manuscrit 24461 mélange les traditions médiévales et des compositions où souffle un esprit prérenaissant. Après les Six Triumphes de Pétrarque, «translatés» en français par Jean Robertet, on trouve tour à tour des figures mythologiques (dieux et déesses antiques, les muses, les Sibylles), des énoncés moraux et des proverbes illustrés, des femmes de différentes nations, l'allégorisation des couleurs et, enfin, quelques femmes célèbres où se perçoit l'influence

35. Les Proverbes illustrés, p. 40 sq.

36. Par exemple le médaillon en l'honneur de François I ${ }^{\text {er }}$ (fol. 138v), après quelques feuillets restés blancs.

37. Hypothèse contestée par F. Buttay-Jutier, Fortuna, p. 336. Mais son argument (l'influence du Liber de ascensu, de 1512), basé sur une seule illustration, est-il décisif? On retrouve le dessin illustrant les «degrez dangereux» de l'ambition aussi dans le Séjour d'Honneur d'Octovien de Saint-Gelais, œuvre dédiée à Charles VIII, mais dont cette copie (BnF, fr. 12783, fol. 153) date de 1508-1510.

38. Date avancée par K. Straub, "Se riens y a qui soit de noble umbrage" ", p. 276.

39. Voir la tapisserie aux armes de la famille Robertet, cat. 159, in France 1500, p. 312.

40. Voir G. Tournoy-Thoen, "Le manuscrit 1010 de la Biblioteca de Cataluña et l'humanisme italien à la cour de France vers 1500 », p. 76, texte n 52. 
des Hérö̈des d'Ovide, qu'Octovien de Saint-Gelais venait de traduire en français à l'intention du roi Charles VIII.

Le manuscrit exprime d'abord l'attachement de la famille Robertet à l'œuvre de Jean, le rhétoriqueur. Il témoigne aussi d'un intérêt certain pour Henri Baude ${ }^{41}$, clerc des finances d'origine bourbonnaise, auteur de pièces de théâtre et de textes satiriques. Bien que son nom ne figure nulle part, certains de ses Dictz moraulx pour faire tapisserie sont intégrés ${ }^{42}$ à la section contenant les dits et proverbes ${ }^{43}$ "en figure» ${ }^{44}$. Ainsi, le quatrain déjà cité, qui évoque les marins séduits par les sirènes, est illustré au fol. 52r. Fortune par contre n'est guère présente ${ }^{45}$ dans les dits de Baude, alors qu'elle hante littéralement les feuillets du recueil Robertet: l'implacable déesse s'invite dans les discours des personnages (fol. 76r, 87r), ailleurs elle est (aussi) représentée dans les dessins (fol. 9r, 55r), le plus souvent identifiable grâce à ses attributs: le bandeau qui lui couvre les yeux, l'arbre ${ }^{46}$ de Fortune (fol. 50r) ou, évidemment, la célèbre roue (fol. $8 \mathrm{v}, 69 \mathrm{r}$ ).

Parmi les représentations du recueil, il y en a une qui ressort particulièrement, car elle est en position d'ouverture, se situant - dans le manuscrit de la Bibliothèque nationale et dans l'exemplaire de l'Arsenal ${ }^{47}$ - immédiatement après les feuillets consacrés aux muses. Or, le dessin, avec lequel commencent les Dits en figure, offre le pendant exact de la peinture au château Saint-Maire (fig. 2). Toutefois, le mouvement du cheval y est inversé: dans le manuscrit, celui-ci court de droite à gauche et non pas de gauche à droite comme dans la salle de l'évêque. Un tel non-respect du sens de la lecture est symboliquement

4I. Voir P. Champion, "Maître Henri Baude, élu des finances et poète» et l'importante notice de J. Blanchard, "Henri Baude».

42. Voir l'édition d'A. Scoumanne, déjà citée, p. 26 sq.

43. Les proverbes strictu sensu se limitent aux fol. 69-97: c'est la partie éditée par E. Rassart-Eeckhout et T. Van Hemelryck.

44. C'est le terme utilisé par le bibliothécaire qui a établi la Table du contenu du manuscrit de l'Arsenal (ms. 5066, fol. Av).

45. Elle figure dans la seule pièce IX de l'édition d'A. Scoumanne.

46. L'attribut, peu habituel, pourrait être un avatar de l'arbre du Paradis (le fruit défendu). Octovien de Saint-Gelais puise à la même source quand il décrit l'arbre de Vaine Espérance avec ses poires aliénantes (Séjour d'Honneur, p. 206-208). Image comparable encore chez Dante: les golosi tendent en vain leurs mains vers les branches d'un pommier chargé de fruits (Purg. XXIV, 103-111).

47. BnF, fr. 24461, fol. 37r; Arsenal, ms. 5066, fol. 37r. 
fort, car il traduit au niveau figuratif la folie de Jeunesse qui agit à l'encontre de ce qu'enseigne la raison. Pourquoi donc ne pas avoir suivi le modèle? L'explication est à chercher, nous semble-t-il, dans l'emplacement qu'occupe la peinture au château. Même si la niche crée un espace à part, le cheval entraîne Jeunesse loin de la seule fenêtre vers le coin le plus sombre de la pièce, là où se dresse le terrible rocher de Fortune. De la lumière à l'obscurité, c'est une autre symbolique qu'exploite habilement l'artiste (sur ordre du commanditaire?); en plus, Jeunesse s'éloigne de la paroi où se trouve l'arbre généalogique des Montfalcon, vers lequel elle retourne pourtant la tête, contrairement à la Jeunesse du manuscrit, qui regarde droit devant elle. Au désordre de la jeunesse s'oppose l'ordre des alliances familiales, expression de la réussite sociale du princeévêque; s'opposent aussi, d'un point de vue stylistique, le registre bas (voir infra) et le registre élevé (la célébration dynastique). On retrouve, au château Saint-Maire, la structure en contrappunto, tel qu'on la connaît d'autres demeures seigneuriales en Europe ${ }^{48}$ : dans le Palazzo del Popolo à San Gimignano (Toscane), les scènes de bon'amor (la vie conjugale) répondent aux scènes de folles amours (les malheurs qui guettent l'homme séduit par une courtisane); au château de La Manta (Piémont), la galerie des neuf preux et des neuf preuses, à la gloire des marquis de Saluces, font face à la Fontaine de Jouvence dont les scènes "carnavalesques", à l'érotisme marqué, rappellent le monde des farces ou des fabliaux.

Le manuscrit nous fournit encore d'autres informations propres à éclairer la peinture du château. Deux inscriptions - absentes ou disparues à Lausanne - précisent que la jeune femme est l'allégorie de la Jeunesse, le cheval celle de la "voulenté", notion qui a ici le sens d'envie ou de désir - excessif ${ }^{49}$, faut-il ajouter. C'est ce que nous avions compris: la scène représente la jeunesse en proie à ce que, dans le vocabulaire aristotélicien de l'époque, on appelle les «appétits». Les deux termes clés figurent également dans le quatrain décasyllabique transcrit au-dessus du dessin:

48. M. L. Meneghetti, Storie al muro, p. 226-242.

49. Comme sous la plume d'Octovien de Saint-Gelais qui utilise, lui aussi, l'image du rocher: "Mais Fortune le fist descendre / Et getta sa nef pour tout voir / En la roche de Trop Vouloir" (Le Séjour d'Honneur, p. 161, v. 38-40). Le Séjour a été composé entre 1491 et 1494. 
Fig. 2 - Jeunesse emportée par le cheval Volonté (Paris, BnF, fr. 24461, fol. 37r). (C) Bibliothèque nationale de France. 
Sur ce cheval qui Voulenté se nomme

Sans bride va Jeunesse l'importune* *qui agit mal à propos

Contre le roch perilleux de Fortune

Ou jadis s'est precipité maint homme ${ }^{50}$.

Même si le message de base se répète du manuscrit au château SaintMaire, ni le texte ni la forme ne se correspondent. Dans le manuscrit, il s'agit d'un quatrain à rimes embrassées $(a b b a)$; à Lausanne, on a opté pour des rimes croisées ( $a b a b$ ), la forme que privilégie la partie des Proverbes en peinture dans le manuscrit. Du point de vue du contenu, la peinture murale se distingue en précisant que le cheval est «sans selle ne sans frain ». La variante est moins anodine qu'il n'y paraît, car elle cache une allusion érotique: "chevaucher sans selle" ${ }^{51}$ est, au passage du Moyen Âge à la Renaissance, une métaphore courante pour désigner l'acte sexuel. Il y a là un aspect ludique indéniable: le quatrain pointe du doigt le triomphe du bas-ventre sur la raison plus qu'il ne déplore l'absence du "joug" si nécessaire à la jeunesse selon la Bible (Lam. 3, 27). Entre empathie et sourire grivois, la peinture du château se distingue d'énoncés comparables par un ton qui lui est propre.

Le dernier vers du manuscrit introduit un élément inconnu au texte lausannois en suscitant l'idée d'une galerie de personnes abattues par Fortune, digne des exemples recueillis dans le De casibus virorum illustrium de Boccace. Il donne ainsi à la leçon une assise à la fois historique et générale, étendant la mise en garde à tout homme. Au château par contre, la folle Jeunesse se retrouve seule sur la sellette. Peutêtre a-t-on jugé qu'il n'était pas nécessaire d'expliciter une leçon que chacun est capable de tirer par lui-même en se demandant en quoi la représentation le concerne. Mais le recentrement sur la figure de Jeunesse accentue le contraste avec les peintures du corridor central où se font face les Vertus de Noblesse et les suivantes de dame Rhétorique, autrement dit les qualités de ceux qui, par l'action ou par l'écriture, façonnent l'histoire. Passer de la niche à l'arbre généalogique de la salle, puis au corridor, c'est passer de la jeunesse - reléguée dans son coin - à l'âge mûr, de l'insouciance à la conscience, créant l'impression d'un vécu,

50. BnF, fr. 24461, fol. 37r.

51. Voir les exemples cités par le Dictionnaire du moyen français (Atilf) en ligne, entrée "chevaucher». 
d'une évolution personnelle. Pour topique que soit le parcours - c'est celui des pèlerinages de vie humaine (voir supra) -, le prince-évêque s'en est servi pour exprimer une leçon qui, s'inscrivant dans l'espace du château, porte la signature de son locataire. Aymon de Montfalcon fait siennes la sagesse proverbiale aussi bien que les valeurs prônées par les textes d'Alain Chartier et de George Chastelain. Les peintures sont l'expression d'une sensibilité personnelle, car elles laissent entrevoir un moi, mais un moi qui, loin de se livrer dans son intimité, se veut exemplaire. Le programme du château Saint-Maire est en fin de compte un manifeste de sagesse, un miroir de perfection: il reflète un idéal de vie, celui qu'a suivi le prince et dont les hôtes et courtisans sont censés s'inspirer à leur tour.

Cet idéal est un idéal partagé. La représentation dans la salle des conférences, tout comme la décoration dans le corridor central, témoignent que les cultures aux cours de Lausanne, de Savoie, de Bourgogne et de Bourbon ont bien des traits en commun. Mais le dit en peinture fait plus qu'offrir une confirmation de ce que historiens, spécialistes de l'art et de la littérature ont mis en évidence depuis un certain temps déjà. La représentation de Jeunesse situe Aymon de Montfalcon dans un cadre plus large, celui d'une élite française dont il partage les goûts. À l'époque, le Breviaire des nobles d'Alain Chartier est largement diffusé en France, voire en Europe ${ }^{52}$; quant à l'intérêt pour les proverbes illustrés, il n'est pas l'apanage de la seule famille Robertet ou du princeévêque. Ainsi, le manuscrit 24461 a directement influencé les peintures du château de Busset dans l'Allier ${ }^{53}$; celles-ci ont été exécutées pour Pierre de Bourbon ( $†$ 1529), neveu de Jean II de Bourbon, le protecteur d'Henri Baude.

Nous restons, il est vrai, toujours dans le même milieu, mais force est de constater que le recueil a circulé - et bien circulé: ses dessins ont aussi inspiré les illustrations de ce joyau qu'est le Petit Livre d'Amour, offert par Pierre Sala à sa seconde épouse ${ }^{54}$, Marguerite Bullioud. Ce notable lyonnais était officier sous Charles VIII, valet de chambre au

52. Voir les contributions de O. Robinson et de A. Kinch dans A Companion to Alain Chartier (c. 1385-1430), surtout p. 247-252 et 289-291.

53. A. Regond, La peinture murale du XVI e siècle dans la région Auvergne, p. 86-98.

54. Voir Pierre Sala, Petit Livre d'Amour, surtout p. 241-247. 
service de Louis XII; retiré des affaires, il conserva son titre d'écuyer sous François I ${ }^{\text {er }}$, qu'il eut l'honneur d'accueillir dans sa demeure de l'Antiquaille en 1522. C'est à la cour de France qu'il aura eu connaissance des Dits et proverbes en figure, soit par l'intermédiaire de François Robertet, soit grâce à son frère, l'influent Florimont ${ }^{55}$, qui était secrétaire des Finances et homme de confiance de François I ${ }^{\text {er }}$. On connaît par ailleurs le goût du roi et de son entourage pour des recueils de petit format contenant des préceptes moraux et politiques, lesquels annoncent la vogue des emblèmes. L'œuvre de François Demoulins de Rochefort ${ }^{56}$, précepteur de François $\mathrm{I}^{\mathrm{er}}$, puis grand aumônier de France, en offre un témoignage éclairant. Sa Vie de la belle et clere Magdalene (1516), composée pour Louise de Savoie et illustrée par Godefroy le Batave ${ }^{57}$ (enlumineur de François $\mathrm{I}^{\mathrm{er}}$ ), comporte une série de médaillons, dans la bordure desquels se trouvent des sentences édifiantes en français, latin, grec ou italien. Comme le quatrain du château Saint-Maire, certaines d'entre elles dénoncent la folie des plaisirs mondains: Volubilia sunt bona mortalium (BnF, fr. 24955, fol. 8r), affirme l'une; Sine dignitate non est venanda voluptas (fol. 10r), enseigne l'autre!

N'est-ce pas justement cette dignité qui manque à Jeunesse emportée, nue, sur un cheval sans bride qu'elle chevauche assise à califourchon, comme le font les hommes? La scène en camaïeu, au cœur du médaillon illustrant la seconde sentence, représente aussi la Madeleine à cheval, mais habillée en femme noble et portant un épervier sur le poignet. Comme il se doit pour une femme respectable, elle chevauche à l'amazone, dans une position instable qui ne lui permet pas de guider le cheval et encore moins de le lancer au galop. La Madeleine dépend de l'écuyer qui tient la monture richement harnachée par la bride, lui indiquant le chemin à suivre... qui est celui de la perdition. La quête du plaisir prend ici la forme d'une dénonciation du paraître (le vêtement, la manière de monter le cheval, l'oiseau de proie), lequel cache l'être véritable de

55. Celui-ci se serait attiré les «faveurs royales en offrant à Madame l'ensemble des Ditz et Proverbes conservé dans le ms. ", affirment E. Rassart-Eeckhout et T. Van Hemelryck, Les Proverbes illustrés, p. 41.

56. Ch. Bonnet, «Paroles et faits exemplaires antiques dans l'œuvre de François Demoulins de Rochefort». Le manuscrit BnF, fr. 24955 est consultable sur le site Gallica de la Bibliothèque nationale de France.

57. Au sujet de cet artiste néerlandais, voir L. de Laborde, "Godefroy, peintre de François I ${ }^{\text {er }}$. 
la personne (le comportement scandaleux de la Madeleine), indigne du statut qu'elle affiche. Le manuscrit et la peinture murale se rejoignent néanmoins en condamnant l'un et l'autre l'empire des sens.

Éthique et esthétique s'allient pour chanter un idéal princier. La cour de France sert de référence; à Paris ou en province, les fonctionnaires d'origine bourgeoise et leurs maîtres baignent dans la même ambiance culturelle. Seigneurs ecclésiastiques, villes et noblesse de robe sont, autour de 1500, un puissant facteur de diffusion artistique ${ }^{58}$, que favorisent les liens personnels. Les artistes et les œuvres circulent, contribuant au rayonnement des centres régionaux. Lettrés au vernis humaniste, ayant chacun passé par l'Italie, Aymon de Montfalcon et Pierre Sala continuent néanmoins à s'inspirer de la littérature médiévale: le premier suit les traces d'Alain Chartier et de Jean de Meun; le second adapte la matière tristanienne et Le Chevalier au lion de Chrétien de Troyes au goût du jour. Les peintures du corridor offrent au public un speculum en l'invitant à lire le Bréviaire des nobles; sous le voile d'un petit livre d'amour, l'ouvrage de Pierre Sala cache une dénonciation des vices curiaux ${ }^{59}$, compréhensible seulement aux gens proches de la cour royale. Un esprit d'époque, celui d'une élite "entre Moyen Âge et Renaissance» - comme le précise le sous-titre de l'exposition France 1500 présentée au Grand Palais à Paris (oct. 2010 / janv. 2011) - flotte décidément au château Saint-Maire de Lausanne. Un esprit qu'incarne à sa manière Jeunesse sur son cheval fou.

\author{
Jean-Claude MüHLethaler \\ Université de Lausanne
}

58. F. Joubert, «Les acteurs de la scène artistique», p. 40-42.

59. Voir P. Cifarelli, «Pierre Sala et le Petit Livre d'Amour (manuscrit Londres, BL, Stowe MS 955)", surtout sa conclusion, p. 76. 


\section{BIBLIOGRAPHIE}

\section{Sources}

Antitus, Poésies, éd. par Manuela Python, Genève, Droz, 1992 (Textes littéraires français, 422) [éd. du mss. ACV, P Antitus, numérisé sous <http://www.e-codices.unifr.ch/en/list/one/acv/P-Antitus >].

Charles d'Orléans, Poetry of Charles d'Orléans and His Circle. A Critical Edition of BnF MS. fr. 25458, Charles d'Orléans's Personal Manuscript, ed. by John Fox, Mary-Jo Arn, Tempe (Arizona), Turnhout, Brepols, 2010 (Arizona Studies in the Middle Ages and the Renaissance, 34).

Christine de Pizan, Le Livre de l'advision Cristine, éd. par Christine Reno, Liliane Dulac, Paris, Champion, 2001.

George Chastelain, Les Exposicions sur Verité mal prise. Le Dit de Verité, éd. par Jean-Claude Delclos, Paris, Champion, 2005.

George Chastelain, Jean Robertet, Jean de Montferrant, Les Douze Dames de Rhétorique, éd. par David Cowling, Genève, Droz, 2002.

Henri Baude, Dictz moraulx pour faire tapisserie, éd. par Annette Scoumanne, Genève/Paris, Droz/Minard, 1959.

Jean Robertet, Euvres, éd. par Margaret Zsuppàn, Genève/Paris, Droz/Minard, 1970.

Michault Taillevent, Un poète bourguignon du XVe siècle: Michault Taillevent, éd. par Robert Deschaux, Genève, Droz, 1975.

Octovien de Saint-Gelais, Le Séjour d'Honneur, éd. par Frédéric Duval, Genève, Droz, 2002.

Pierre Sala, Petit Livre d'Amour (Stowe MS 922, British Library), commentaire de Janet Backhouse, Yves Giraud, Lucerne, Faksimile Verlag, 1994.

Les Proverbes illustrés, éd. par Emmanuelle Rassart-Eeckhout, Tania Van Hemelryck, Montréal, Ceres, 2001. 
Le Roman de la Rose, éd. et trad. par Armand Strubel, Paris, Le Livre de Poche (Lettres gothiques), 1992.

\section{Travaux}

Blanchard, Joël, "Henri Baude», in Écrivains juristes et juristes écrivains du Moyen Âge au siècle des Lumières, éd. par Bruno Méniel, Paris, Classiques Garnier, 2015, p. 600-603.

Bonnet, Charlotte, «Paroles et faits exemplaires antiques dans l'œuvre de François Demoulins de Rochefort: réminiscences et réemplois des figures antiques à l'aube du règne de François $\mathrm{I}^{\mathrm{er}}$ ", in Actualiser le passé: figures antiques du Moyen Âge à la Renaissance, éd. par Jean-Claude Mühlethaler, Delphine Burghgraeve, Lausanne, Centre d'études médiévales et post-médiévales, 2012, p. 184-196 (en ligne: <https://serval.unil.ch/notice?pid=serval:BI B_34DBDAE31C15>).

Bousmar, Éric, "Faut-il restituer Antitus Favre à la littérature bourguignonne? Connections austro-bourguignonnes dans la carrière et l'œuvre d'un rhétoriqueur peu connu († entre 1501 et 1506)", in "A l'heure encore de mon escrire». Aspects de la littérature de Bourgogne sous Philippe le Bon et Charles le Téméraire, éd. par Claude Thiry, Louvain-la-Neuve, Les Lettres Romanes, 1997, p. 9-22.

Bove, Boris, «Réflexions sur les hommes nouveaux et l'ascension sociale au Moyen Âge, de Leudaste à Jacques Cour, en passant par Pareto", in Hommes nouveaux et femmes nouvelles, de l'Antiquité au XX $X^{e}$ siècle, éd. par Benoît Musser, Rennes, Presses universitaires de Rennes, 2015, p. 37-57.

Buttay-Jutier, Florence, Fortuna. Usages politiques d'une allégorie morale à la Renaissance, Paris, Presses universitaires ParisSorbonne, 2008.

Buzzetti Gallarati, Silvia (a cura di), Jean de Meun, Testamento e Codicillo. Etica, cultura, politica nella Parigi medievale, Fiesole, Nardini, 1996.

Champion, Pierre, "Maître Henri Baude, élu des finances et poète», in Histoire poétique du quinzième siècle, Paris, Champion, 1923, vol. 2, p. 239-307 
Cifarelli, Paola, "Pierre Sala et le Petit Livre d'Amour (manuscrit Londres, BL, Stowe MS 955)", in Le recueil au Moyen Âge. La fin du Moyen Âge, éd. par Benoît Musser, Turnhout, Brepols, 2010, p. 61-77.

Doudet, Estelle, Poétique de George Chastelain (1415-1475). "Un cristal mucié en un coffre", Paris, Champion, 2005.

FÉRY-HüE, Françoise, "Aymon de Montfalcon", in Dictionnaire des lettres françaises: le Moyen Âge, dir. par Geneviève Hasenohr, Michel Zink, Paris, Fayard, 1992, p. 119-120.

Foenr-Janssens, Yasmina, "La Maison de Fortune dans l'Anticlaudianus d'Alain de Lille", in La Fortune. Thèmes, représentations, discours, éd. par Yasmina Foehr-Janssens, Emmanuelle Métry, Genève, Droz, 2003, p. 129-144.

France 1500. Entre Moyen Âge et Renaissance, catalogue de l'exposition au Grand Palais, 6 octobre 2010-10 janvier 2011, Paris, Éditions de la Réunion des Musées Nationaux, 2010.

HüE, Denis, "L’orgueil du cheval», Senefiance, 32 (1992: Le cheval dans le monde médiéval), p. 257-276.

Joubert, Fabienne, «Les acteurs de la scène artistique», in France 1500. Entre Moyen Âge et Renaissance, catalogue de l'exposition au Grand Palais, 6 octobre 2010-10 janvier 2011, Paris, Éditions de la Réunion des Musées Nationaux, 2010, p. 38-44.

Jung, Marc-René, "Les "Douze Dames de Rhétorique" ", in Du mot au texte. Actes du III colloque international sur le moyen français, éd. par Peter Wunderli, Tübingen, Narr, 1982, p. 229-240 (Tübinger Beitrage zur Linguistik, 175).

Kinch, Ashby, "Chartier's European Influence", in A Companion to Alain Chartier (c. 1385-1430). Father of French Eloquence, ed. by Daisy Delogu, Joan E. McRae, Emma Cayley, Leiden/Boston, Brill, 2015, p. 279-302.

LABORDe, Léon de, "Godefroy, peintre de François $\mathrm{I}^{\mathrm{er}}$ ", Revue universelle des arts, 1 (1855), p. 5-18.

Maupeu, Philippe, Pèlerins de vie humaine. Autobiographie et allégorie narrative, de Guillaume de Deguileville à Octovien de Saint-Gelais, Paris, Champion, 2009.

Meneghetti, Maria Luisa, Storie al muro. Temi e personaggi della letteratura profana nell'arte medievale, Torino, Einaudi, 2015. 
Mühlethaler, Jean-Claude, Fauvel au pouvoir: lire la satire médiévale, Paris, Champion, 1994.

—, «Le tyran à table. Intertextualité et référence dans l'invective politique à l'époque de Charles VI", in Représentation, pouvoir et royauté à la fin du Moyen Âge, éd. par Joël Blanchard, Paris, Picard, 1995, p. 49-62.

—, "Quand Fortune, ce sont les hommes. Aspects de la démythification de la déesse, d'Adam de la Halle à Alain Chartier», in La Fortune. Thèmes, représentations, discours, éd. par Yasmina Foehr-Janssens, Emmanuelle Métry, Genève, Droz, 2003, p. 177-206.

—, «Ein dichterisches und politisches Manifest in Lausanne. Zur Interpretation des Breviaire des Nobles und der Douze Dames de Rhétorique in den Fresken des Schlosses Saint-Maire aus literaturhistorischer Sicht", in Paroles de murs / Sprechende Wände, éd. par Eckart Conrad Lutz, Dominique Rigaux, Grenoble, 2007, p. 91-105 (Cahiers du Centre de recherche en histoire et histoire de l'art. Italie, pays alpins, 10).

Mühlethaler, Jean-Claude, Burghgraeve, Delphine (dir.), Un territoire à géographie variable. La communication littéraire au temps de Charles VI, Paris, Classiques Garnier, 2017.

Prévot, Brigitte, Ribémont, Bernard, Le cheval en France au Moyen Agge, Orléans, Paradigme, 1994.

Regond, Annie, La peinture murale du XVI e siècle dans la région Auvergne, Clermont-Ferrand, Institut d'Études du Massif Central, 1983.

RibÉmont, Bernard, "Le regard de Christine de Pizan sur la jeunesse (à propos du Charles V)", Cahiers de Recherches Médiévales et Humanistes, 7 (2000), p. 255-260 (<http://journals.openedition. org/crm/904>).

Robinson, Olivia, "Alain Chartier: The Manuscript and Print Tradition", in A Companion to Alain Chartier (c. 1385-1430). Father of French Eloquence, ed. by Daisy Delogu, Joan E. McRae, Emma Cayley, Leiden/Boston, Brill, 2015, p. 223-252.

Rochat, Lise, Les peintures du corridor du château Saint-Maire à Lausanne, Mémoire d'histoire de l'art moderne, sous la direction du Prof. Mauro Natale, Université de Genève, 2005 (inédit).

Straub, Karen, " "Se riens y a qui soit de noble umbrage, / Qui soit de fruit ou de cler fenestrage / Pour decorer royal palais ou porge 
/ Soit pris en gré" ", in Poètes et artistes. La figure du créateur en Europe au Moyen Âge et à la Renaissance, éd. par Sophie CassagnesBrouquet, Martine Yvernault, Limoges, Presses universitaires de Limoges, 2007, p. 273-288.

-, "Les Douze Dames de Rhétorique" in Text und Bild, Affalterbach, Didymos, 2016.

Tervarent, Guy de, Attributs et symboles dans l'art profane, Genève, Droz, 1997.

Tournoy-Thoen, Godelieve, "Le manuscrit 1010 de la Biblioteca de Cataluña et l'humanisme italien à la cour de France vers 1500 ", Humanistica Lovaniensa: Journal of Neo-Latin Studies, 26 (1977), p. 1-81. 
